\title{
Protocolo de Cuidado Farmacêutico a Indivíduos com Diabetes Mellitus
}

\author{
Protocol of Pharmaceutical Care for Individuals with Diabetes Mellitus \\ Protocolo de Atención Farmacéutica a Individuos con Diabetes Mellitus
}

Gabriel Silas Borges Silva de Melo ${ }^{1 *}$, Sabrina Rodrigues Ribeiro ${ }^{1}$, Amanda Silva de Sousa ${ }^{1}$, Beatriz Scarleth Noleto de Souza ${ }^{1}$, Alessandra Camillo da Silveira Castello Branco'.

\section{RESUMO}

Objetivo: Elaborar uma proposta de Protocolo Clínico de Cuidado Farmacêutico para Indivíduos com Diabetes Mellitus. Métodos: $O$ presente trabalho foi realizado na forma de pesquisa qualitativa, com caráter descritivo para elaboração do protocolo clínico seguindo a Metodologia Dáder. As informações científicas foram coletadas utilizando bancos de dados na internet como Periódicos Capes e Biblioteca Virtual em Saúde (BVS). Foram selecionados artigos científicos do período de 2007 a 2019. Resultados: O Protocolo de Cuidado Farmacêutico proposto foi elaborado segundo a Metodologia Dáder de Seguimento Farmacoterapêutico, pegando como base informações sobre o Diabetes Mellitus, com o intuito de montar uma ferramenta competente que auxilie no tratamento, adesão do paciente, e fornecer orientações ao mesmo. Conclusão: Pode-se concluir que o Protocolo Clínico de Cuidado Farmacêutico, contém informações e orientações importantes acerca da doença e do tratamento, a fim de identificar e prevenir os problemas relacionados aos medicamentos, que incluem as reações adversas e as interações medicamentosas. Contudo, espera-se que esta proposta de protocolo de cuidado venha a auxiliar os profissionais farmacêuticos, durante o serviço de seguimento farmacoterapêutico de indivíduos com Diabetes Mellitus, trazendo benefícios à saúde da população.

Palavras-chave: Protocolo, Cuidado Farmacêutico, Diabetes Mellitus.

\begin{abstract}
Objective: To elaborate a proposal of Clinical Protocol of Pharmaceutical Care for Individuals with Diabetes Mellitus. Methods: The present work was carried out in the form of qualitative research, with descriptive character for elaboration of the clinical protocol following the Dáder Methodology. Scientific information was collected from using Internet databases such as Periódicos Capes and Biblioteca Virtual em Saúde (BVS). Current scientific papers (2007-2019 period) were selected. Results: The Protocol of Pharmaceutical Care was developed according to the Dáder Methodology of Pharmaco-therapeutic Follow-up, based on information about Diabetes Mellitus, with the purpose of assembling a competent tool that assists in the treatment, adherence of the patient, and provide guidelines for it. Conclusion: It can be concluded that the Clinical Protocol for Pharmaceutical Care contains important information and guidance about the disease and treatment to identify and prevent drug-related problems, which include adverse reactions and drug interactions. However, it is expected that this proposal of care protocol will help the pharmaceutical professionals, during the service of pharmacotherapeutic follow-up of individuals with Diabetes Mellitus, seeking to support this process, bringing benefits to the health of the population.
\end{abstract}

Key words: Protocol, Pharmaceutical Care, Diabetes Mellitus.

${ }^{1}$ Centro Universitário Santo Agostinho (UNIFSA), Teresina-PI. *E-mail: gabrielsilasborges@gmail.com

SUBMETIDO EM: 4/2019

ACEITO EM: 5/2019

PUBLICADO EM: $8 / 2019$

REAS/EJCH | Vol.Sup.29 | e843 | DOI: https://doi.org/10.25248/reas.e843.2019 Página 1 de 7 


\section{RESUMEN}

Objetivo: Elaborar una propuesta de Protocolo Clínico de Cuidado Farmacéutico para Individuos con Diabetes Mellitus. Métodos: El presente trabajo fue realizado en la forma de investigación cualitativa, con carácter descriptivo para la elaboración del protocolo clínico siguiendo la Metodología Dáder. Las informaciones científicas fueron recolectadas utilizando bases de datos en Internet como Periódicos Capes y Biblioteca Virtual en Salud (BVS). Se seleccionaron artículos científicos del período de 2007 a 2019. Resultados: El Protocolo de Cuidado Farmacéutico propuesto fue elaborado según la Metodología Dáder de Seguimiento Farmacoterapéutico, tomando como base informaciones sobre la Diabetes Mellitus, con el propósito de montar una herramienta competente que auxilie en el tratamiento, la adhesión del paciente, y proporcionar orientaciones al mismo. Conclusión: Se puede concluir que el Protocolo Clínico de Cuidado Farmacéutico, contiene informaciones y orientaciones importantes sobre la enfermedad y el tratamiento, a fin de identificar y prevenir los problemas relacionados con los medicamentos, que incluyen las reacciones adversas y las interacciones medicamentosas. Sin embargo, se espera que esta propuesta de protocolo de cuidado venga a auxiliar a los profesionales farmacéuticos durante el servicio de seguimiento farmacoterapéutico de individuos con Diabetes Mellitus, aportando beneficios a la salud de la población.

Palabras clave: Protocolo, Cuidado Farmacéutico, Diabetes Mellitus.

\section{INTRODUÇÃO}

Os protocolos clínicos são ferramentas essenciais durante o acompanhamento farmacoterapêutico de pacientes acometidos principalmente por doenças crônicas. Consistem em formulários ou fichas, utilizadas por exemplo na Metodologia Dáder de acompanhamento farmacoterapêutico, nas quais o farmacêutico irá preencher manualmente, anotando dados sobre a situação atual do paciente, relacionando a doença e a farmacoterapia. Isto é, são documentos utilizados para construir o perfil farmacoterapêutico, durante o serviço de atenção farmacêutica (HERNÁNDEZ DS et al., 2009).

Esses protocolos são extremamente relevantes na atenção à saúde, pois atuam como suporte na disponibilização de procedimentos e no gerenciamento da atenção farmacêutica. Trata-se de um instrumento de coleta de dados sobre a história patológica e terapêutica do paciente, que permite ao farmacêutico um estudo aprofundado e permite que ele avalie a necessidade, segurança e efetividade da terapia (MINISTÉRIO DA SAÚDE, 2014).

Além disso, a elaboração e execução de protocolos clínicos, auxiliam a fazer o diagnóstico de doenças, identificar Problemas Relacionados aos Medicamentos (PRM), definir um esquema de farmacoterapia, fazer orientações e esclarecer dúvidas do paciente. Estas ações promovem o uso racional dos medicamentos e o autocuidado, onde o farmacêutico pode fazer intervenções quando necessário, para a obtenção de resultados terapêuticos positivos (PEREIRA LRL e DE FREITAS O., 2008).

É comum a utilização desses formulários no acompanhamento de doenças crônicas, principalmente no cuidado ao Diabetes Mellitus (DM), tendo em vista que esta é uma das doenças crônicas mais comuns no mundo. Segundo estudo da Federação Mundial de Diabetes - IDF (2014), o número estimado de diabetes no mundo no ano de 2013 era de 387 milhões de pessoas, onde 46\% delas não apresentavam diagnóstico prévio. Em relação aos países da América do Sul e América Central, a estimativa era de 24 milhões de pessoas acometidas. A projeção para o ano de 2035 seria de 38,5 milhões para esses países, representando um aumento de $60 \%$ nos casos.

Trata-se de uma doença metabólica, onde o organismo tem problemas na secreção ou na ação do hormônio insulina, produzido pelo pâncreas. Esses defeitos são consequências de problemas genéticos ou podem aparecer com o passar do tempo. Dentre os tipos mais comuns desta doença têm-se o Diabetes tipo I (DM1), que é quando o pâncreas fornece pouca ou nenhuma insulina para poder metabolizar a glicose, e o Diabetes tipo II (DM2), que é quando a forma como o corpo processa a glicose no sangue é afetada. 
O tratamento desta patologia está ligado ao controle glicêmico aliado a uma boa dieta alimentar e prática de exercícios físicos regulares, a fim de se obterem valores mais próximos da normalidade (TEIXEIRA ABM, 2017).

No que tange a incidência do DM1, dados da Sociedade Brasileira de Diabetes (SBD) mostram que o número de casos diagnosticados vem aumentando nas últimas décadas, principalmente em crianças com menos de 5 anos. Ademais, apresenta taxas de 7,6 no Brasil por 100 mil indivíduos com idade inferior a 15 anos. Com o DM2 é um pouco diferente, tendo em vista que por ser o que mais acomete a população mundial, este é dito como decorrente da maturidade. É difícil, portanto, estabelecer a incidência deste tipo em grandes populações pois demanda muito tempo com aferição de glicemia periódica. Porém, segundo pesquisas também da SBD, nos últimos anos têm-se observado um crescimento no número de casos de DM2 em adolescentes (SBD, 2017).

Esse crescimento desordenado no número de casos de DM devem-se a mudança no estilo de vida, como o sedentarismo, alimentação inadequada e estresse. Esses fatores somados podem levar ao surgimento de outros problemas de saúde como a obesidade, hipertensão e hipercolesterolemia. Então, é importante manter o foco na análise do estilo de vida atual da população, com o intuito de procurar recursos em saúde e comunitários para neutralizar a prevalência desta patologia (SHAW JE et al., 2010).

Apesar dos avanços em relação à terapia do DM, este continua sendo um enorme problema de saúde, principalmente porque a população possui dificuldade em manter o controle glicêmico, evidenciando resultados insatisfatórios, que necessitam ser acompanhados. Nesse contexto, o farmacêutico pode auxiliar através do acompanhamento farmacoterapêutico, utilizando-se protocolos clínicos de cuidado, uma ferramenta importante para a segurança, qualidade e eficácia do tratamento. Diante disso, essa pesquisa tem como objetivo elaborar uma proposta de Protocolo Clínico de Cuidado Farmacêutico para Indivíduos com Diabetes Mellitus.

\section{MÉTODOS}

O presente trabalho foi realizado na forma de pesquisa qualitativa, com caráter descritivo para elaboração do protocolo clínico seguindo a Metodologia Dáder, que é um método de acompanhamento farmacoterapêutico que visa obter resultados positivos na saúde do paciente. Para isso, as informações científicas foram coletadas através dos bancos de dados: Periódicos Capes e Biblioteca Virtual em Saúde (BVS). Foram selecionados artigos científicos do período de 2007-2019, utilizando os seguintes descritores: Protocolo Clínico, Diabetes Mellitus e Metodologia Dáder, sendo excluídos aqueles que não se enquadravam nos objetivos do estudo.

As informações coletadas foram transcritas para o programa Microsoft Word, para construção do protocolo de cuidado farmacêutico, de acordo com a Metodologia Dáder de Seguimento Farmacoterapêutico. Considerando os parâmetros dessa pesquisa, que inclui a coleta de informações científicas para estruturação da ferramenta que se propõe, os riscos são considerados mínimos. Quanto aos benefícios, estes envolvem a elaboração de um protocolo mais eficaz e que auxilie numa melhor adesão do paciente ao tratamento, além de fornecer orientações ao indivíduo acerca da doença e do uso correto dos medicamentos.

\section{RESULTADOS E DISCUSSÃO}

O Protocolo de Cuidado Farmacêutico proposto foi elaborado segundo a Metodologia Dáder de Seguimento Farmacoterapêutico, pegando como base informações sobre o Diabetes Mellitus (conceito, etiologia, classificação, epidemiologia, diagnóstico e tratamento), com o intuito de montar uma ferramenta competente que auxilie no tratamento, adesão e autocuidado do indivíduo (Apêndice Suplementar).

A Metodologia Dáder foi um método criado por um grupo de farmacêuticos espanhóis da Universidade de Granada, na Espanha, em 1999. Foi utilizado em muitos países para ajudar na execução do serviço de atenção farmacêutica. Objetiva avaliar o paciente acometido com determinada doença, podendo ser realizado 
em qualquer âmbito assistencial, de forma sistematizada, continuada e documentada. Para isso, os dados são coletados para construir a história farmacoterapêutica, para que a partir dela se compreenda a situação do doente, permitindo visualizar o panorama sobre a sua saúde e o seu tratamento em momentos distintos, assim como avaliar os resultados da farmacoterapia (BISSON MP, 2007; HERNÁNDEZ DS et al., 2009).

Este método se baseia na construção do perfil farmacoterapêutico, pegando como base a história clínica do paciente, ou seja, todos os problemas de saúde enfrentados pelo paciente bem como os medicamentos que ele utiliza. Com isso, o farmacêutico pode identificar e resolver os Problemas Relacionados aos Medicamentos (PRM), além de promover intervenções para resolver esses problemas e para que possa também avaliar posteriormente os resultados obtidos, diminuindo, então, os Resultados Negativos da Medicação (RNM) (MENESES ALL e SÁ MLB, 2010).

No Terceiro Consenso de Granada, um evento criado para discutir sobre o Seguimento Farmacoterapêutico, foi definido que os problemas de saúde são entendidos como resultados clínicos negativos devidos à farmacoterapia. Neste evento foi proposta uma lista de PRM's que podem ser apontadas como possíveis causas de um RNM, dentre essas PRM's listadas pode-se destacar a administração errada do medicamento, conservação inadequada, contraindicação, erros na dispensação, erros na prescrição, interações, problemas de saúde insuficientemente tratados e outros. Neste consenso também se estabeleceu uma classificação dos RNM's em função dos requisitos que todo medicamento deve ter para ser utilizado: que seja necessário, efetivo e seguro (Quadro 1) (CARRILHO JRF, 2013).

Quadro 1 - Classificação de Resultados Negativos da Medicação, segundo Terceiro Consenso de Granada.

\begin{tabular}{|c|l|}
\hline \multirow{3}{*}{ Necessidade } & $\begin{array}{l}\text { Problema de saúde não tratado. O doente sofre de um problema } \\
\text { de saúde associado ao fato de não receber a medicação que } \\
\text { necessita. }\end{array}$ \\
\hline & $\begin{array}{l}\text { Efeito de medicamento não necessário. O doente sofre de um } \\
\text { problema de saúde associado ao fato de receber um } \\
\text { medicamento de que não necessita. }\end{array}$ \\
\hline \multirow{5}{*}{ Efetividade } & $\begin{array}{l}\text { Efetividade não quantitativa. O doente sofre de um problema de } \\
\text { saúde associado a uma inefetividade não quantitativa da } \\
\text { medicação. }\end{array}$ \\
\hline & $\begin{array}{l}\text { Efetividade quantitativa. O doente sofre de um problema de } \\
\text { saúde associado a uma inefetividade quantitativa da medicação. }\end{array}$ \\
\hline \multirow{5}{*}{ Segurança } & $\begin{array}{l}\text { Insegurança não quantitativa. O doente sofre de u0m problema } \\
\text { de saúde associado a uma insegurança não quantitativa de um } \\
\text { medicamento. }\end{array}$ \\
\hline & $\begin{array}{l}\text { Insegurança quantitativa. O doente sofre de um problema de } \\
\text { saúde associado a uma insegurança quantitativa de um } \\
\text { medicamento. }\end{array}$ \\
\hline
\end{tabular}

Fonte: CARRILHO JRF, 2013.

O acompanhamento farmacêutico, segundo a Metodologia Dáder, se dá início com a procura deste serviço pelo paciente acometido por alguma patologia e quando há necessidade por parte dele. Na primeira entrevista, ao ofertar o serviço, o farmacêutico registra todos os dados pessoais do paciente, para que se crie uma espécie de prontuário para ele. Depois, registra-se também suas queixas principais, os hábitos alimentares, os medicamentos e as terapias alternativas nas quais faz uso (NUNES PHC et al., 2008).

No protocolo proposto (Apêndice Suplementar), criou-se espaços para registro de todos os dados pessoais importantes, para contato, identificação e montagem do perfil do paciente. A data da primeira consulta e a data do atendimento, incluídas nesse formulário são importantes para que se tenha um controle do tempo em que o paciente está em observação. E o número de prontuário seria importante para uma busca mais ágil do perfil do paciente, além de permitir a continuidade dos cuidados prestados ao indivíduo. 
Uma área específica para história social do paciente também foi construída, tendo em vista que é importante saber se há outras pessoas que convivem com o paciente e que o auxiliam durante o tratamento. Ademais, é relevante procurar entender o histórico da doença, se deve a um fator hereditário ou não, pois isso facilita na compreensão sobre o diagnóstico do paciente. O uso de bebidas alcóolicas e outras drogas, bem como a prática de atividades físicas são informações necessárias, pois interferem na terapia do indivíduo.

Como as taxas glicêmicas são as mais afetadas devido o defeito no metabolismo, o tratamento do DM é voltado para o controle dessas taxas, com a finalidade de obter-se valores mais próximos possíveis da normalidade, e isso se alcança através da utilização medicamentos orais ou insulina para o controle glicêmico, aliado a uma boa dieta alimentar e prática de exercícios físicos regulares (BOAS LCGV et al., 2011).

Para o tratar o DM, é comum o médico propor classe de hipoglicemiante oral que mais irá se adequar ao estado situacional do paciente, a fim de promover uma melhor adesão do indivíduo ao tratamento. Dentre essas classes, pode-se citar, segundo seu mecanismo de ação principal: aqueles que melhoram a secreção de insulina (sulfonilureias e glinidas); os que diminuem a velocidade de absorção de glicídios (inibidores das alfaglicosidases); os que reduzem a produção hepática de glicose (biguanidas); e por fim, os que aumentam a utilização periférica de glicose (glitazonas) (SBD, 2017).

Então, aliado à terapia medicamentosa, é muito significativo que o profissional farmacêutico proponha mudanças comportamentais ao paciente, com a finalidade de prevenir complicações agudas e crônicas. Além de negociar prioridades, as orientações fornecidas motivam o paciente a cuidar da sua saúde. Como o tratamento depende muito do empenho do indivíduo, manter o controle metabólico por muito tempo torna-se complicado, então a atenção ao paciente e o monitoramento da sua adesão ao tratamento é essencial (GIROTTO E et al., 2013)

Para isso, criou-se uma área somente para registro dos hábitos alimentares, a fim de documentar e posteriormente avaliar os hábitos de vida, e como eles podem interferir no tratamento deste paciente. Neste campo, poderão ser descritos, conforme o relato do paciente, os alimentos nos quais faz uso de acordo com cada refeição do dia, além do horário em que o mesmo faz esta refeição. Logo abaixo têm-se também as terapias alternativas ou complementares que o indivíduo também faz uso, como acupuntura, fitoterápicos, homeopáticos e remédios caseiros, estes também pelo mesmo motivo necessitam ser documentados.

Nos protocolos clínicos as informações mais importantes e que necessitam ser documentadas são as queixas e complicações do paciente durante a terapia. Tendo em vista, que isso permite que o farmacêutico identifique quais as frustrações, ou ainda o motivo da não adesão ao tratamento. No campo referente as queixas, pode-se registrar qual o problema enfrentado, sinais, sintomas, tempo de início, frequência, duração e exames relacionados ao problema relatado, ou seja, é uma área específica para as queixas sobre a doença ou outras patologias associadas.

Logo em seguida têm-se outro campo para registro dos problemas com a medicação, onde o paciente pode relatar qual medicamento mas lhe incomoda, além de poder avaliar em uma escala de 0 a 10 o grau de incomodo. Em relação as dificuldades enfrentadas pelo paciente frente a embalagem, aquisição e utilização dos medicamentos, tem-se um espaço para descrever o grau de dificuldade em muito, pouco ou nada, além de poder-se anotar qual medicamento provoca esta dificuldade. No campo sobre complicações, 0 farmacêutico pode avaliar qual o nível de informação o paciente possui sobre o diabetes, como por exemplo: se ele sabe a como tratar a hiperglicemia, se ele tem conhecimento sobre as principais complicações, sinais e sintomas da doença, bem como as medidas que o paciente toma frente a isso.

Posteriormente, tem-se de fato o local onde o farmacêutico irá anotar todo e qualquer medicamento que 0 paciente faz uso, quem prescreveu, a indicação, apresentação e posologia, se faz uso correto do medicamento e a data de início e a duração do tratamento.

Após essa primeira entrevista, o farmacêutico pode marcar uma nova data para retorno do paciente, e com todas essas informações ele irá avaliar o que foi relatado e documentado para em seguida fazer a intervenção seja ela com o próprio paciente, ou com o médico caso o erro seja proveniente da prescrição. 
Após analisar todos os aspectos narrados pelo paciente sobre a suas queixas e farmacoterapia, é feita a análise da fase situacional do paciente, e realiza-se uma fase de estudo, onde há a identificação dos PRM's e prováveis Resultados Negativos da Medicação (RNM). Em seguida, o farmacêutico decidirá a necessidade de realizar a intervenção farmacêutica, tanto com o paciente ou com o médico, dependendo da ocorrência de RNM. Podem ser marcadas várias entrevistas para acompanhar se houve evolução nos resultados (BISSON MP, 2007).

Durante o processo de análise, há dois campos exclusivos do farmacêutico no formulário, que é a avaliação e intervenção. No campo sobre avaliação farmacêutica, o profissional irá anotar todos os medicamentos, avaliar a necessidade, efetividade e segurança de cada um, e é neste momento que ele poderá identificar os RNM's, que podem ser: interações medicamentosas, apresentação errada, dose errada, ou ainda se o medicamento em uso está provocando reações adversas responsáveis pela queixa relatada pelo paciente. Com isso, no campo de intervenção farmacêutica, o profissional irá descrever a solução para os problemas identificados.

No retorno do paciente, o farmacêutico irá repassar as intervenções ao paciente. E nas consultas subsequentes, é que de fato o farmacêutico poderá visualizar se houve evolução no quadro do paciente, anotando no campo exclusivo para consultas subsequentes, se ele apresenta uma nova queixa, poderá descrever um pouco a história clínica, as mudanças feitas nos hábitos de vida (dieta e atividades físicas) e classificação de risco. Também há um espaço somente para registro dos exames clínicos e laboratoriais. Tudo isso é crucial para efeito de comparação e elucidação do quadro clínico do indivíduo. É importante ressaltar, que no retorno e nas entrevistas subsequentes, após análise e intervenção, o farmacêutico pode fazer orientações sobre o uso correto e racional dos medicamentos, indicar melhores hábitos de vida, esclarecer dúvidas pertinentes a doença e terapia medicamentosa, ou seja, promovendo o autocuidado e a educação em saúde, pois são artifícios que garantem a adesão ao tratamento.

A educação em saúde quando envolve abordagens comportamentais e comtempla aspectos sociais, culturais e cognitivos, viabiliza a construção dos conhecimentos e reflexão sobre a doença. Consiste em uma estratégia pedagógica no processo de intervenção no estilo de vida dos indivíduos com DM, o que certifica a adesão ao tratamento e estimula o autocuidado (TORRES HC et al., 2011).

\section{CONSIDERAÇÕES FINAIS}

Pode-se concluir que o Protocolo Clínico de Cuidado Farmacêutico, contém informações e orientações importantes acerca da doença e do tratamento, a fim de identificar e prevenir os problemas relacionados aos medicamentos, que incluem as reações adversas e as interações medicamentosas. Além disso, é um instrumento que visa promover o uso racional das medicações e a educação em saúde, garantir a eficácia do tratamento, em benefício de assegurar a adesão do paciente ao tratamento e o autocuidado, melhorando assim a qualidade de vida da população. Contudo, espera-se que esta proposta de protocolo de cuidado venha auxiliar os profissionais de saúde, principalmente os farmacêuticos, durante o serviço de seguimento farmacoterapêutico de indivíduos com Diabetes Mellitus, a fim de trazer benefícios à saúde da população.

\section{REFERÊNCIAS}

1. BISSON MP. Farmácia clínica \& atenção farmacêutica. Manole, 2007.

2. BRASIL. Ministério da Saúde. Secretaria de Atenção à Saúde. Protocolos clínicos e diretrizes terapêuticas: volume 3. Brasília: Ministério da Saúde, 2014; 604p.

3. BOAS LCGV, et al. Adesão à dieta e ao exercício físico das pessoas com diabetes mellitus. Texto \& ContextoEnfermagem, 2011; 20(2): 272-279.

4. CARRILHO JRF. Adequação do Método Dáder no seguimento farmacoterapêutico em doentes hipertensos institucionalizados num lar de idosos. Dissertação (Mestrado em Ciências Farmacêuticas) - Ciências da Saúde. Universidade da Beira Interior, Covilhã, 2013; 127 p. 
5. GIROTTO E, et al. Adesão ao tratamento farmacológico e não farmacológico e fatores associados na atenção primária da hipertensão arterial. Ciência \& Saúde Coletiva, 2013; 18(1): 1763-1772.

6. HERNÁNDEZ DS, et al. Método Dáder: Manual de seguimento farmacoterapêutico. Universidade de Granada. Tradução: Inês Isabel Lopes Nunes da Cunha. Brasil, 2009.

7. INTERNATIONAL DIABETES FEDERATION. IDF Diabetes Atlas. 6th ed. Brussels: International Diabetes Federation; 2014.

8. MENESES ALL, SÁ MLB. Atenção farmacêutica ao idoso: fundamentos e propostas. Geriatria \& Gerontologia, 2010; 4(3): 154-161.

9. NUNES PHC, et al. Intervenção farmacêutica e prevenção de eventos adversos. Revista Brasileira de Ciências Farmacêuticas, 2008; 44(4): 691-699.

10. PEREIRA LRL, DE FREITAS O. A evolução da Atenção Farmacêutica e a perspectiva para o Brasil. Revista Brasileira de Ciências Farmacêuticas, 2008; 44(4): 601-612.

11. SHAW JE, et al. Global estimates of the prevalence of diabetes for 2010 and 2030. Diabetes research and clinical practice, 2010; 87(1): 4-14.

12. SOCIEDADE BRASILEIRA DE DIABETES. Diretrizes da Sociedade Brasileira de Diabetes (2017-2018). São Paulo: Editora Clannad, 2017; 383p.

13. TEIXEIRA ABM, et al. Educação em saúde para o autocuidado e o controle glicêmico aos portadores de diabetes mellitus em uma Unidade de Saúde de Curitiba-PR. Anais do EVINCI-UniBrasil, 2018; 3(1): 186-186.

14. TORRES HC, et al. Intervenção educativa para o autocuidado de indivíduos com diabetes mellitus. Acta paul enferm, $2011 ; 24(4): 514-519$. 